\title{
Tailoring GMI effect in Co-rich glass coated microwires by Joule heating
}

\author{
Karin García ${ }^{1}$, Arcady Zhukov ${ }^{1}$, Valentina Zhukova ${ }^{2}$, Manuel Provencio ${ }^{1}$, Alexandr Torcunov ${ }^{2}$ \\ Manuel Vazquez ${ }^{1}$, Marek Kuźmiński ${ }^{3}$, Julian Gonzalez ${ }^{4}$ and Henryk Lachowicz ${ }^{3}$. \\ ${ }^{1}$ Instituto de Ciencia de Materiales, CSIC, 28049 Cantoblanco, Madrid, Spain \\ 2 "TAMag Iberica" S.L., Avda. de Remedios 41-3", Colmenar Viejo, Madrid, Spain. \\ ${ }^{3}$ Institute of Physics, Polish Academy of Sciences, Al.Lotników 32/46, 02-668 Warszawa, Poland \\ ${ }^{4}$ Dpto. Fis. Mater., Fac. Química, UPV/EHU, P.O. Box 1072, 20080, San Sebastián, Spain
}

The dependence of magnetoimpedance ratio, $\Delta Z / Z=$ $\left\{\left[Z(H)-Z\left(H_{\max }\right)\right] / Z\left(H_{\max }\right)\right\} \cdot 100 \%$, on the axial magnetic field of soft magnetic $\mathrm{Co}_{67} \mathrm{Fe}_{3.85} \mathrm{Ni}_{1.45} \mathrm{~B}_{11.5} \mathrm{Si}_{14.5} \mathrm{Mo}_{1.7}$ glass coated amorphous microwires has been investigated in the frequency range $1-30 \mathrm{MHz}$, for the driving current amplitudes $0.75-5 \mathrm{~mA}$, and at the maximum axial DC-field of $8 \mathrm{kA} / \mathrm{m}$. In the as-prepared sample, maximum GMI ratio, $\Delta Z / Z_{\max } \approx 380 \%$, has been observed at the highest used frequency $(30 \mathrm{MHz})$. It has, however, been shown, that Joule heating enables to enlarge the $\Delta Z / Z_{\max }$ ratio up to $500 \%$. A strong dependence of $\Delta Z / Z_{\max }$ on annealing time, frequency and amplitude of the driving current has also been observed. The results of the GMI measurements, presented as a function of the annealing time with the frequency and current amplitude as the parameters, showed an increase of the anisotropy field with an increase of the annealing time and temperature. The observed dependencies have been interpreted in terms of stress relaxation and changes of the magneto-elastic anisotropy induced by the Joule heating.

Key words: glass-coated microwires, giant magnetoimpedance, thermal treatment

\section{Introduction}

The giant magnetoimpedance (GMI) effect had become a phenomenon of a great interest for sensor applications owing to the high sensitivity of the impedance to the applied $D C$-field (up to $300 \%$ in conventional amorphous wires). Such changes of the impedance of a magnetic conductor have been interpreted in terms of the classical skin effect, as a consequence of the change of the penetration depth of an $A C$-current flowing through a magnetic conductor and caused by the applied $D C$-field ${ }^{1)}$. Enhanced GMI ratio in magnetically soft amorphous wires was explained by a specific domain structure in the outer domain shell exhibiting high circumferential permeability 1), 2). Presently existing strong tendency of miniaturization of magnetic elements has resulted in the development of the Taylor-Ulitovsky method, which permits to produce tiny ferromagnetic metallic wires $(3-30 \mu \mathrm{m}$ in diameter) covered by an insulating glass coating ${ }^{3)}$. Recent significant progress in tailoring glass coated microwires fabricated by this method enabled to enlarge significantly the GMI ratio (up to about $600 \%)^{4)-6 \text { ). }}$

A comparison of the magnetic field dependence of the $\Delta Z / Z_{\max }$-ratio with the magneto-optical Kerr effect (MOKE) hysteresis loops proved that the maximum of the GMI ratio appears at the $D C$-magnetic field at which the bamboo-like circular domain structure appears in the outer shell of the wire. Such a structure is created when the axial $D C$-field decreases from the saturation value to zero ${ }^{7)}$. Both, bulk hysteresis loops and GMI effect are strongly affected by the geometry of the samples. This fact is utilised to tailor both, magnetic softness of the wire and its GMI effect (changing glass coating thickness, in particular). Such dependence of both magnetic and transport properties of glass coated microwires of the same composition is related to the magnetoelastic contribution appearing from the difference in the thermal expansion coefficients of metallic core and glass coating. Therefore, it seems to be reasonable to assume, that the heat treatment significantly affects the internal stress distribution, which results in significant changes of both, domain structure and the GMI effect behaviour. of glass coated microwires

In this paper we report the results of tailoring the GMI ratio of amorphous $\mathrm{Co}_{67} \mathrm{Fe}_{3.85} \mathrm{Ni}_{1.45} \mathrm{~B}_{11.5} \mathrm{Si}_{14.5} \mathrm{Mo}_{1.7}$ microwires by Joule heating.

\section{Experimental details}

Magnetically soft, glass-coated $\mathrm{Co}_{67} \mathrm{Fe}_{3.8 .5} \mathrm{Ni}_{14.5} \mathrm{~B}_{11.5}$ $\mathrm{Si}_{14.5} \mathrm{Mo}_{1.7}$ microwires of different geometric ratio, $\rho$, of metallic core diameter to total microwire diameter $0.789 \leq \rho$ $\leq 0.98$ and exhibiting low magnetic anisotropy field (50$200 \mathrm{~A} / \mathrm{m}$, see Fig.1) has been fabricated by the Taylor-Ulitovsky method ${ }^{3)-6)}$. The sample geometry, i.e. average values of the diameter of the metallic core, $d$, total diameter, $D$ and the ratio, $\rho=d / D$, measured at several positions along the sample are presented in Table 1 . The hysteresis loops of the samples have been measured by conventional fluxmetric method. The electrical impedance of the microwire was evaluated by means of the four-point technique. The magneto- impedance ratio, $\Delta Z / Z$, has been defined as: 
Table 1. The sample geometrical characteristics

\begin{tabular}{|c|c|c|c|}
\hline Sample & $\begin{array}{c}\text { Metallic core } \\
\text { diameter, } d(\mu \mathrm{m})\end{array}$ & $\begin{array}{c}\text { Total diameter, } \\
D(\mu \mathrm{m})\end{array}$ & $\begin{array}{c}\text { Ratio } \\
\rho=d / D\end{array}$ \\
\hline $\mathrm{A}$ & 22.4 & 22.8 & 0.982 \\
\hline $\mathrm{B}$ & 21.4 & 26.2 & 0.817 \\
\hline $\mathrm{C}$ & 22.4 & 28.4 & 0.789 \\
\hline
\end{tabular}

$\Delta Z / Z=\left[Z(H)-Z\left(H_{\max }\right)\right] / Z\left(H_{\max }\right)$,

An axial $D C$-field of its intensity up to $8 \mathrm{kA} / \mathrm{m}$ was supplied by a Helmholtz coils. The dependence of the magnetoimpedance ratio, $\Delta Z / Z$, on the axial field, $H$, at the driving $A C$-current amplitude, $I$, ranging from 0.75 up to 5 $\mathrm{mA}$ of the frequency, $f$, in the range $1-30 \mathrm{MHz}$, both treated as the parameters, have been investigated. using computer controlled system designed in the Institute of Physics, Polish Academy of Science.

Thermal treatment has been realized by the Joule heating of the sample when a $D C$-current of 30 and $40 \mathrm{~mA}$ flows along the microwire-sample, with various heating times of this treatment. Electrical contacts were made removing mechanically the glass coating at the sample edges and soldering them with the $\mathrm{Cu}$ cables.

\section{Results and discussion}

Measured bulk hysteresis loops of three magnetically soft, glass-coated $\mathrm{Co}_{67} \mathrm{Fe}_{3.85} \mathrm{Ni}_{1.45} \mathrm{~B}_{11.5} \mathrm{Si}_{14.5} \mathrm{Mo}_{1.7}$ microwires of various geometric ratio $0.78 \leq \rho \leq 0.98$ are shown in Fig.1. As it can be observed in this figure, the anisotropy field, $H_{k}$ increases with decreasing $\rho$-ratio, i.e. with an increase of the glass coating thickness. On the other hand, the field $H_{m}$, corresponding to the maximum of the GMI ratio, increases and $(\Delta Z / Z)_{\max }$ decreases with a decrease of the $\rho$-ratio, as it is seen in Fig.2. Such a tendency allows to assume that the parameter $\rho$ is the important parameter, which affects both, the hysteretic and GMI features. Therefore, soft magnetic properties and GMI effect can by tailored by the sample geometry. It means that the internal stresses, arising from the difference between the thermal expansion coefficients of the metallic core and glass coating, strongly affects the aforementioned properties. Therefore, the relaxation of such internal stresses by means thermal treatment should drastically change both, soft magnetic behaviour and $\triangle Z / Z(H)$ dependence. This challenge is of a special importance since the fabrication of the microwires with the glass coating thickness of about 0.2 $\mu \mathrm{m}$ (sample A) is a difficult task. This is why from the view-point of applications it is very important to identify the processing procedure improving the GMI effect of glass coated microwires. The effect of Joule heat treatment was performed using the sample B (see Table 1). As expected, the performed Joule heating strongly affects the $\triangle Z / Z(H)$ dependence. Fig. 3 shows this dependence measured for as-prepared (i.e. not treated) and annealed samples with the frequency, $f$, as a parameter. It is well

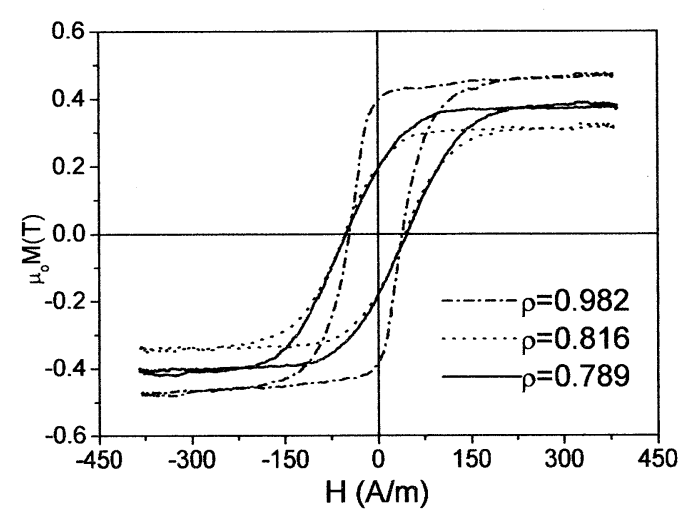

Fig.1. Hysteresis loop of three samples with $\rho$ as a parameter

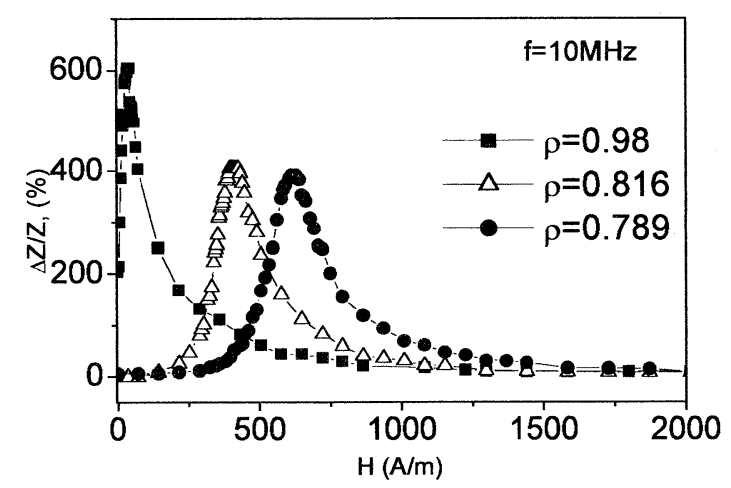

Fig.2. Axial field dependence of $\Delta Z / Z$ at $f=10 \mathrm{MHz}$ and $I=0.75 \mathrm{~mA}$ in microwire with $\rho$ as a parameter.

recognized from the Fig. 3 that the maximum value of the GMI ratio, $\Delta Z / Z_{\max }$, increases after Joule heating. On the other hand the value of the axial $D C$-field, $H_{m}$, corresponding to the maximum of the GMI ratio, increases also in the samples subjected to the Joule heating (see Fig.3).

In order to illustrate better the effect of the Joule heating only, the $\Delta Z / Z(H)$ dependencies have been measured at the same conditions, i.e. at the fixed frequency and driving current amplitude, varying solely the Joule heating conditions.

These dependencies are presented in Fig.4a (annealing current $30 \mathrm{~mA})$ and in Fig. $4 \mathrm{~b}(40 \mathrm{~mA})$.

Another important parameter affecting the $\Delta Z / Z(H)$ dependencies is the driving $A C$-current amplitude, $I$. Fig.5 shows the effect of the driving current amplitude on the GMI behaviour of the as-prepared and annealed micro-wires.

In this way there are few parameter which permit to tailor the GMI effect of glass coated microwires, such as time, $t_{a n n}$, and $D C$ current during the Joule heating, frequency, $f$, and driving $A C$-current amplitude, $I$, during the measurements of the GMI effect. The effect of all these parameters on the maximum GMI ratio, $\Delta Z / Z_{\max }$, is summarized in the Fig.6. 

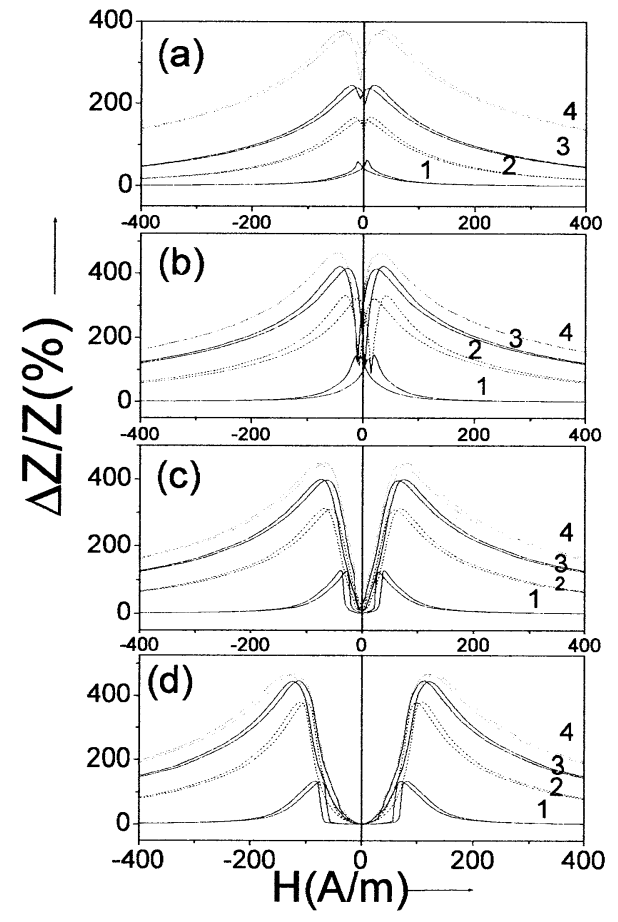

Fig.3. $\triangle Z / Z(H)$ dependences measured at $I=1 \mathrm{~mA}$ in as-prepared microwire (a) annealed at $30 \mathrm{~mA}$ for 2 minutes (b) at $40 \mathrm{~mA}$ for 2 minutes (c) and at $40 \mathrm{~mA}$ for 10 minutes (d) for $f=1 \mathrm{MHz}$ (1), $10 \mathrm{MHz}$ (2), $20 \mathrm{MHz}$ (3)and $30 \mathrm{MHz}$ (4).

It is worth to notice that not only the maximum of the GMI ratio, $\Delta Z / Z_{\max }$, but also the shape of $\Delta Z / Z(H)$ dependences change under the effect of the current annealing. In particular, the field corresponding to the maximum of the GMI ratio, $H_{m}$, depends on the current annealing conditions. Fig. 7 summarizes the effect of all the mentioned parameters on $H_{m}$.

Experimental dependencies observed at various samples geometry and conditions of current annealing can be attributed to the effect of internal stresses, $\sigma$, on the domain structure as well as to the magnetic anisotropy. Indeed, the value of the axial $D C$-field, $H_{\mathrm{m}}$ at which the GMI-ratio achieves maximum corresponds to the static circular

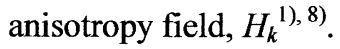

The estimated values of the internal stresses in these amorphous microwires are of the order of $1000 \mathrm{MPa}$, depending strongly on the thickness of glass coating and metallic core diameter ${ }^{9)}$. Such large internal stresses give rise to a drastic change of the magnetoelastic energy, $K_{m e} \approx$ $3 \lambda_{s} \sigma_{i} / 2$, even for small changes of the glass-coating thickness at fixed metallic core diameter. Consequently, such change of the $\rho$-ratio should be related to the change of the magnetostriction constant ${ }^{8)}$ :

$$
\lambda_{\mathrm{s}}=\left(\mu_{\mathrm{o}} M_{\mathrm{s}} / 3\right)\left(\mathrm{d} H_{\mathrm{k}} / \mathrm{d} \sigma\right)
$$

where $\mu_{\mathrm{o}}$ is the free space permeability equals $4 \pi \times 10^{-7}$ $\mathrm{H} / \mathrm{m}$ and $M_{s}$ is the saturation magnetization. In fact, general

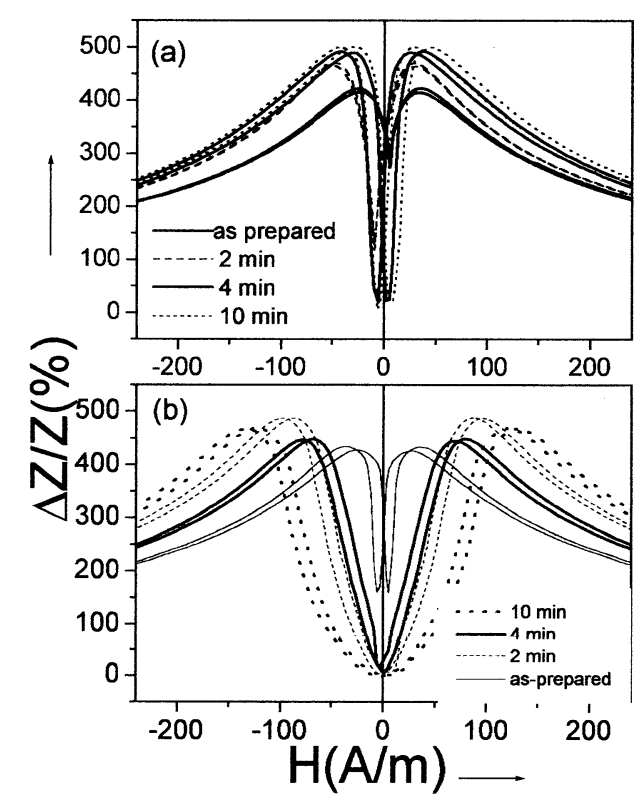

Fig. 4. $\triangle Z / Z(H)$ dependences measured at $f=30 \mathrm{MHz}$ and $I=1 \mathrm{~mA}$ in microwire annealed at $30 \mathrm{~mA}(\mathrm{a})$ and at $40 \mathrm{~mA}(\mathrm{~b})$.

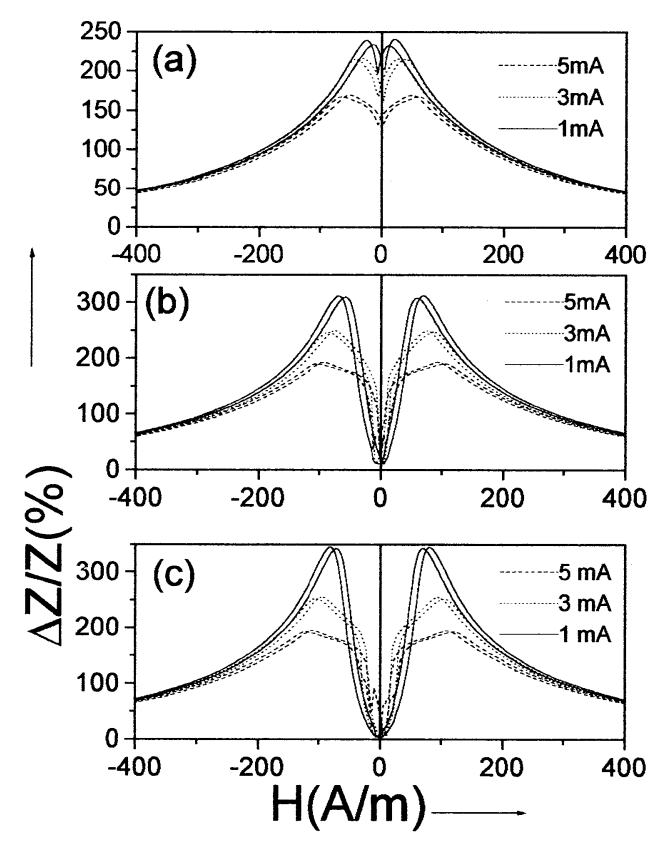

Fig. 5. Effect of driving current amplitude on $\Delta Z / Z(H)$ dependences measured at $f=10 \mathrm{MHz}$ in as-prepared (a), annealed at $40 \mathrm{~mA}$ for $2 \mathrm{~min}$ (b) and at $40 \mathrm{~mA}$ for 4 min (c) microwires.

increase of the $H_{m}$ value with annealing time has been observed (see Fig.7), which in according to (2) should be attributed to the increasing of the magnetic anisotropy field with $t_{a n n}$. Taking into account the phenomenologically found ${ }^{10)}$ stress dependence of the magnetostriction

$$
\lambda_{\mathrm{s}}(\sigma)=\lambda_{\mathrm{s}}(0)-A \sigma
$$




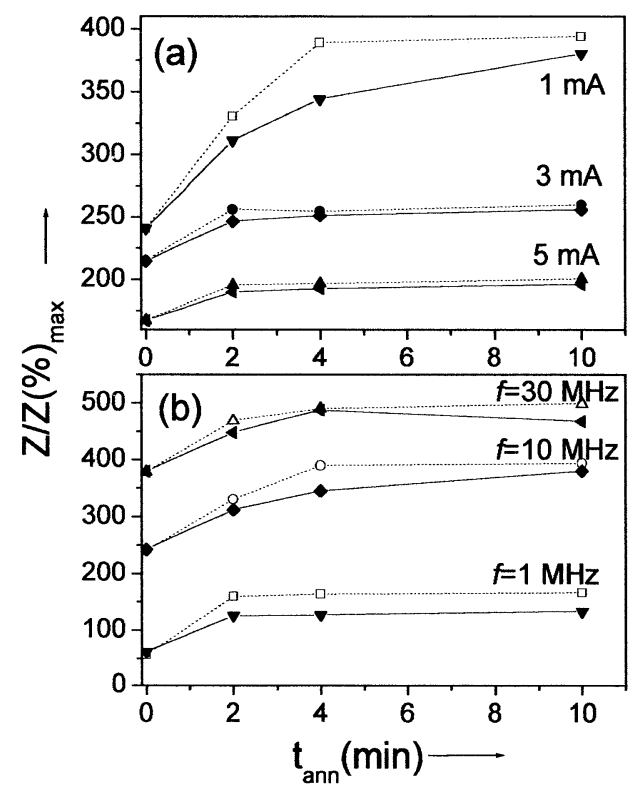

Fig. 6. Effect of driving current amplitude (measurements performed at $f=30 \mathrm{MHz}$ ) (a)and frequency (measurements at $I=1 \mathrm{~mA}$ ) (b) on $\Delta Z / Z_{\max }$ measured in microwires annealed at $30 \mathrm{~mA}$ (dot lines) and at $40 \mathrm{~mA}$ (solid lines) .

where $\lambda_{\mathrm{s}}(0)$ is the saturation magnetostriction constant without applied stresses and $A$ is the positive coefficient of the order of $10^{-10} \mathrm{MPa}$, stress relaxation should provide a decrease of the $H_{m}$ value. Such contradiction can be attributed to the induction of the circular magnetic anisotropy due to the effect of the circular magnetic field created by the $D C$ current during the Joule heating.

On the other hand, relaxation of the internal stresses should also change the shape of the $\Delta Z / Z(H)$ dependencies (particularly, $H_{m}$ value) as well as the value of the $\Delta Z / Z_{\text {max }}$ -ratio.

Alternatively, the observed $\triangle Z / Z(H)$ dependencies vs. driving $A C$-current amplitude and frequency should be related to the magnetization process in the circumferential direction.

\section{Conclusions}

It is demonstrated that the Joule heating of amorphous $\mathrm{Co}_{67} \mathrm{Fe}_{3.85} \mathrm{Ni}_{1.45} \mathrm{~B}_{11.5} \mathrm{Si}_{14.5} \mathrm{Mo}_{1.7}$ microwire can significantly change the $\triangle Z / Z(H)$ dependencies and significantly improve the $\Delta Z / Z_{\max }$ ratio (from 380 to $500 \%$ ). Substantial dependence of $(\Delta Z / Z)(H)$ on frequency and amplitude of the driving $A C$-current, $D C$ bias current and geometric ratio, $\rho$, has also been observed. The shape of the experimental dependencies obtained for various sample geometry and

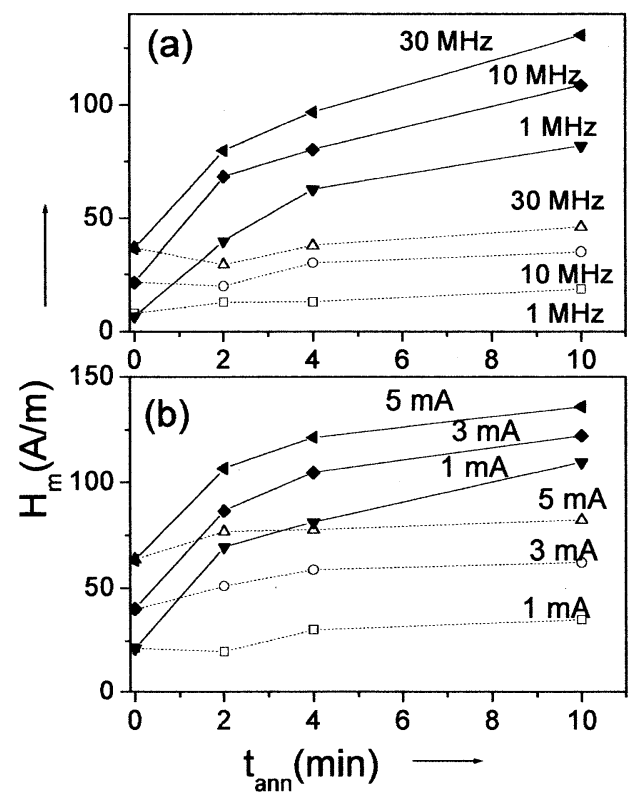

Fig. 7. Effect of frequency $(I=1 \mathrm{~mA})$ (a) and driving $A C$-current amplitude $(f=30 \mathrm{MHz})(\mathrm{b})$ on $H_{m}$ measured in microwires annealed at $30 \mathrm{~mA}$ (dot lines) and at $40 \mathrm{~mA}$ (solid lines).

conditions of current annealing are most probably attributed

to the effect of internal stresses, $\sigma$, on the domain structure as well as to the magnetic anisotropy.

\section{References}

1) L.V. Panina and K. Mohri, Appl. Phys. Lett. 65, 1189 (1994).

2) R.L. Sommer and C.L. Chien, J. Appl. Phys. 79, 5139 (1996).

3) A. F. Cobeño, A. Zhukov, J. M. Blanco and J. Gonzalez, J. Magn. Magn. Mat 234, L359 ( 2001).

4) A. Zhukov, J. Gonzalez, J.M. Blanco, M.J. Prieto, E. Pina and M. Vázquez, J. Appl. Phys. 87, 1402 (2000).

5) L. Kraus, Z. Frait, K. Pirota and H. Chiriac, J. Magn. Magn. Mat 254-255, 399 (2003).

6) A. Zhukov, J. Magn and Magn. Mater. 242-245, 216 (2002).

7) V. Zhukova, A. Chizhik, A. Zhukov, A. Torcunov, V. Larin and J. Gonzalez, IEEE Trans. Magn. 38, 3090 (2002).

8) M. Knobel, C. Gómez-Polo and M. Vázquez, J. Magn. Magn. Mat. 160, 243 (1996).

9) J. Velázquez, M. Vazquez and A. Zhukov, J. Mater. Res. 11 2499 (1996).

10) J.M. Barandiaran, A. Hernando, V. Madurga, O.V. Nielsen, M. Vázquez and M. Vázquez - López, Phys. Rev. 35, 5066 (1987), also A.Siemko, H.K.Lachowicz and B.Lisowski, Acta Phys.Polon.A, 72, 197 (1987).

( Received May 8, 2003; Accepted October 29, 2003 ) 